\title{
C-Reactive Protein and Peripheral Artery Disease among Japanese Elderly: the Tsurugaya Project
}

\author{
Atsushi HOZAWA, Kaori OHMORI, Shinichi KURIYAMA, Taichi SHIMAZU, Kaijun NIU*, \\ Aya WATANDO ${ }^{* *}$, Satoru EBIHARA**, Toshifumi MATSUI ${ }^{* *}$, Masataka ICHIKI $^{* * *}$, \\ Ryoichi NAGATOMI* ${ }^{*}$ Hidetada SASAKI ${ }^{* *}$, and Ichiro TSUJI
}

\begin{abstract}
We investigated the cross-sectional relationship between ankle brachial index and cardiovascular disease risk factors, including C-reactive protein (CRP), among Japanese elderly, a topic which has had little prior epidemiologic study. Our study population comprised 946 subjects aged at least 70 years in whom both CRP and ankle brachial index were measured. The participants were classified into a low (ankle brachial index $<0.9$ ) and normal ankle brachial index group. We found that current smoking, high-density lipoprotein cholesterol $<\mathbf{4 0} \mathrm{mg} / \mathrm{dl}$, a low body mass index (continuous variable), hypertension, diabetes and statin use were all significantly related to a lower ankle brachial index. Higher log-transformed CRP level was significantly related to a lower ankle brachial index after adjustment for the cardiovascular risk factors mentioned above $(p<0.01)$. The odds ratios for low ankle brachial index compared to $0-1$ risk factors were $5.79(95 \%$ confidence interval [Cl]: 2.99-11.20) for 2 risk factors and 17.45 (95\% Cl: 6.78-49.91) for 3 or more risk factors; independently of other risk factors, the odds ratio for CRP $>1.0 \mathrm{mg} / \mathrm{l}$ was 2.10 (95\% Cl: 1.13-3.88) compared to lower CRP values. Thus, a high level of CRP is related to a low ankle brachial index among Japanese elderly as well as Western subjects. This is the first study to report the relationship between CRP and low ankle brachial index among Japanese elderly. (Hypertens Res 2004; 27: 955-961)
\end{abstract}

Key Words: C-reactive protein, cardiovascular risk factors, ankle brachial index, Japanese, elderly

\section{Introduction}

In recent years, C-reactive protein (CRP) has become established as a risk factor for cardiovascular diseases (1-14). Higher levels of CRP predict future myocardial infarction and stroke independently of other cardiovascular disease risk factors, and it has been suggested that the measurement of $\mathrm{CRP}$, in addition to cardiovascular disease risk factors, may improve our ability to predict cardiovascular diseases $(10,13)$.

Peripheral artery disease (PAD) is a severe atherosclerotic condition causing intermittent claudication and is associated with higher incidence of future cardiovascular and cerebrovascular diseases (15-19). The low ankle brachial systolic blood pressure index (ABI) has been used as a measure of lower limb PAD (20). In Western countries, some prospective studies have demonstrated a positive relationship between $\mathrm{CRP}$ and low $\mathrm{ABI}(21,22)$ as well as a relationship

From the Department of Public Health and Forensic Medicine and ${ }^{*}$ Division of Medicine and Science in Sports and Exercise and ${ }^{* *}$ Division of Geriatric and Respiratory Medicine, Department of Tohoku University Graduate School of Medicine, Sendai, Japan, and ${ }^{* * *}$ JR Sendai Hospital, Sendai, Japan.

This study was supported by a Grant-in-Aid for Scientific Research (13557031), by a grant for JSPS Research (14010301) from the Ministry of Education, Culture, Sports, Science and Technology of Japan, by a Research Grant $(2002,2003)$ from the Japan Atherosclerosis Prevention Fund, by a Health Science Grant on Health Services (H13-kenko-008), and by grants for Comprehensive Research on Aging and Health (H13-choju-007, H13choju-023) from the Ministry of Health, Labour and Welfare of Japan.

Address for Reprints: Atsushi Hozawa, M.D., Ph.D., Department of Public Health, Graduate School of Medicine, Tohoku University, 2-1 Seiryomachi, Aoba-ku, Sendai 980-8575, Japan. E-mail: hozawa@mail.tains.tohoku.ac.jp

Received June 28, 2004; Accepted in revised form August 25, 2004. 
between CRP and cardiovascular diseases (1-14).

In Japan, however, epidemiological data about risk factors for low ABI among Japanese have been limited $(23,24)$. Furthermore, no studies have investigated the relationship between CRP and low ABI. Therefore, in the present study, we investigated the relationship between ABI and cardiovascular disease risk factors, including CRP, among Japanese elderly.

\section{Methods}

\section{Study Participants}

Our study population comprised subjects aged 70 years and older who were living in the Tsurugaya area of Sendai, one of the major cities in the Tohoku area of Japan. At the time of the study, there were 2,730 people aged 70 years and older living in Tsurugaya $(25,26)$. We invited all of these individuals to participate in a comprehensive geriatric assessment, which included medical status, physical function, cognitive function and dental status, and 1,178 of these people agreed to participate and give their informed consent for analysis of the data. The protocol for this study was approved by the Institutional Review Board of Tohoku University Graduate School of Medicine. We excluded subjects whose CRP had not been measured $(n=29)$ and subjects whose ABI had not been measured $(n=21)$. We assessed hypertension using home blood pressure (BP) data, and subjects who did not measure their BP on at least 3 days during the 4 -week study period were excluded $(n=176)$. This criterion was based on our previous observation that the average BP values for the first 3 days did not differ significantly from those obtained during the entire study period (27). Furthermore, we excluded subjects who did not complete the questionnaire about alcohol consumption $(n=6)$. Therefore, the study population comprised 946 subjects (mean age $75.2 \pm$ 4.6 years, men: $45 \%$ ).

\section{CRP Measurement}

We collected the blood sample under non-fasting conditions. Serum CRP levels were determined using an immunotechnique on a Behring BN II analyzer (Dade Behring, Tokyo, Japan). The BN II high sensitivity assay utilizes a monoclonal antibody coated on polystyrene particles and fixed-time kinetic nephelometric measurements (28). The BN II nephelometer makes a 1:400 dilution to measure CRP concentrations between 3.5 and $210 \mathrm{mg} / \mathrm{l}$. The assay has been approved by the US Food and Drug Administration for use in assessing the risk of cardiovascular and peripheral vascular disease.

\section{ABI Measurement}

Bilateral ABI was measured in all subjects using a new de- vice, the FORM ABI/PWV (Colin Co., Komaki, Japan), which incorporates an automatic oscillometer (29). The FORM ABI/PWV is a device with four cuffs that can measure BP levels simultaneously in both arms and both legs, and automatically calculates the ABI. This device is useful for mass medical examinations and population-based studies because it enables measurements of ABI and brachial ankle pulse wave velocity in a short time and is not affected by the operator's technique. This device has been used in other Japanese epidemiological studies $(24,30,31)$.

\section{Classification of Subjects}

We treated the lowest $\mathrm{ABI}$ in either leg as the ABI value. We defined the subjects with an $\mathrm{ABI}<0.90$ as the "low ABI" subjects, and we classified serum CRP levels into three groups, $<1 \mathrm{mg} / 1,1$ to $2.9 \mathrm{mg} / \mathrm{l}$ and $3 \mathrm{mg} / \mathrm{l}$ and over, according to the previous reports $(10,13)$.

\section{Data Analysis}

Variables were compared by the $\chi^{2}$ test, $t$-test or analysis of variance, as appropriate. The odds ratio (OR) of PAD was calculated using multiple logistic regression analysis.

We used the following variables as confounding factors: age, sex, smoking habit, drinking habit, hypertension, hypercholesterolemia, a low level of high density lipoprotein (HDL) cholesterol, body mass index (BMI), diabetes, prior cardiovascular diseases and use of statin drugs.

Subjects were considered hypertensive if their home systolic BP (SBP) was at least $135 \mathrm{mmHg}$ and/or home diastolic $\mathrm{BP}$ (DBP) was at least $85 \mathrm{mmHg}$, or if they were using antihypertensive agents $(32,33)$. Subjects were considered diabetic if their non-fasting blood glucose level was at least $200 \mathrm{mg} / \mathrm{dl}$, or if they currently used antidiabetic medication. Subjects were considered hypercholesterolemic if their level of total cholesterol was at least $220 \mathrm{mg} / \mathrm{dl}$, or they currently used non-statin lipid-lowering agents. Low HDL cholesterol was defined as a level of HDL cholesterol below $40 \mathrm{mg} / \mathrm{dl}$. The information on smoking status, drinking status and history of prior cardiovascular diseases was obtained using questionnaire surveys. Current drinkers were also asked about drinking frequency, beverage types usually consumed, and amount consumed on a single occasion. From these responses we calculated the average daily alcohol consumption in grams. Since statins have been reported to lower CRP levels $(34,35)$, we treated them as independent confounding factors. When we analyzed the relationship between low $\mathrm{ABI}$ and $\mathrm{CRP}$ as a continuous variable, we used the logtransformed value (CRP value +1 ), because the CRP distribution was skewed to the right among Japanese (36); we added 1 before transformation because the log-transformation expands the scale for values below 1. Since the CRP level has been reported to be related to risk clustering (37), we analyzed the relationship between low ABI and a combi- 
Table 1. Association between Lower Ankle Brachial Index and Cardiovascular Disease Risk Factors, for 946 Subjects, the Tsurugaya Project, Sendai, Japan, 2002

\begin{tabular}{|c|c|c|c|}
\hline & \multicolumn{3}{|c|}{ Ankle brachial index } \\
\hline & $<0.9$ & $\geqq 0.9$ & $p$ \\
\hline Number of subjects & 54 & 892 & \\
\hline Age (years) & 77 & 76 & $0.049^{*}$ \\
\hline Sex (male \%) & 67 & 43 & $<0.01^{* *}$ \\
\hline Current smoker (\%) & 26 & 12 & $<0.01^{* *}$ \\
\hline Ex-smoker $(\%)$ & 43 & 30 & \\
\hline Never smoker $(\%)$ & 31 & 58 & \\
\hline Mean alcohol consumption (g) & 14 & 10 & $0.37^{*}$ \\
\hline Body mass index $\left(\mathrm{kg} / \mathrm{m}^{2}\right)$ & 24 & 24 & $0.46^{*}$ \\
\hline Hypertension $(\%)$ & 91 & 69 & $<0.01^{* *}$ \\
\hline Diabetes $(\%)$ & 26 & 9 & $<0.01^{* *}$ \\
\hline Hypercholesterolemia (\%) & 31 & 36 & $0.49^{* *}$ \\
\hline Low HDL cholesterol (\%) & 33 & 11 & $<0.01^{* *}$ \\
\hline Use of statin drugs $(\%)$ & 30 & 16 & $0.01^{* *}$ \\
\hline History of cardiovascular diseases (\%) & 31 & 15 & $<0.01^{* *}$ \\
\hline \multicolumn{4}{|c|}{$\begin{array}{l}{ }^{*} t \text {-test, }{ }^{* *} \chi^{2} \text {-test. Hypertension: home systolic blood pressure } \\
\text { (BP) was at least } 135 \mathrm{mmHg} \text { and/or home diastolic BP was at } \\
\text { least } 85 \mathrm{mmHg} \text {, or they were using antihypertensive agents. Dia- } \\
\text { betes: non-fasting blood glucose level was at least } 200 \mathrm{mg} / \mathrm{dl} \text {, or } \\
\text { if they currently used antidiabetic medication. Hypercholes- } \\
\text { terolemia: level of total cholesterol was at least } 220 \mathrm{mg} / \mathrm{dl} \text {, or } \\
\text { they currently used non-statin lipid-lowering agents. Low HDL } \\
\text { cholesterol: level of high density lipoprotein cholesterol below } \\
40 \mathrm{mg} / \mathrm{dl} \text {. }\end{array}$} \\
\hline
\end{tabular}

nation of cardiovascular disease risk factors and CRP level. In this analysis, we treated hypertension, diabetes, current smoking or low HDL cholesterol as cardiovascular disease risk factors.

The drug information was confirmed by an experienced pharmacist. The level of statistical significance was set at $p<0.05$. All statistical analyses were performed with SAS software, version 8.2 (SAS Institute, Cary, USA).

\section{Results}

\section{Association between $\mathrm{ABI}$ and Atherosclerosis Risk Factors}

Table 1 shows the association between low ABI and cardiovascular disease risk factors. The mean age was significantly higher in subjects with low ABI than those without low ABI. The proportions of never smokers and females were lower in low ABI subjects. Similarly, the proportions of subjects with hypertension, diabetes, and low HDL cholesterol, and the proportions of statin users or subjects with a history of prior cardiovascular diseases, were higher in low ABI subjects. The proportions of subjects with hypercholesterolemia did not differ between subjects who had a low ABI and subjects
Table 2. Association between C Reactive Protein (CRP) and Cardiovascular Disease Risk Factors

\begin{tabular}{lrrrc}
\hline & \multicolumn{3}{c}{ CRP (mg/l) } & \multirow{2}{*}{$p$} \\
\cline { 2 - 4 } & -0.9 & $1.0-2.9$ & $3.0-$ & \\
\hline Number of subjects & 637 & 201 & 108 & \\
Age (years) & 76 & 76 & 76 & $0.70^{*}$ \\
Sex (male \%) & 43 & 47 & 47 & $0.44^{* *}$ \\
Current smoker (\%) & 11 & 17 & 12 & $0.02^{*}$ \\
Ex-smoker (\%) & 29 & 32 & 40 & \\
Never smoker (\%) & 60 & 51 & 47 & \\
Alcohol consumption (g) & 11 & 13 & 7 & $0.22^{*}$ \\
Body mass index (kg/m²) & 24 & 25 & 25 & $<0.01^{*}$ \\
Hypertension (\%) & 68 & 74 & 81 & $0.01^{* *}$ \\
Diabetes (\%) & 8 & 11 & 16 & $0.03^{* *}$ \\
Hypercholesterolemia (\%) & 33 & 42 & 40 & $0.047^{* *}$ \\
Low HDL cholesterol (\%) & 11 & 14 & 16 & $0.20^{* *}$ \\
Use of statin drugs (\%) & 18 & 13 & 19 & $0.27^{* *}$ \\
History of cardiovascular & 15 & 17 & 24 & $0.051^{* *}$ \\
diseases (\%) & & & & \\
\hline
\end{tabular}

${ }^{*}$ ANOVA, ${ }^{* *} \chi^{2}$-test. Hypertension: home systolic blood pressure (BP) was at least $135 \mathrm{mmHg}$ and/or home diastolic BP was at least $85 \mathrm{mmHg}$, or they were using antihypertensive agents. Diabetes: non-fasting blood glucose level was at least 200 $\mathrm{mg} / \mathrm{dl}$, or if they currently used antidiabetic medication. Hypercholesterolemia: level of total cholesterol was at least $220 \mathrm{mg} / \mathrm{dl}$, or they currently used non-statin lipid-lowering agents. Low HDL cholesterol: level of high density lipoprotein cholesterol below $40 \mathrm{mg} / \mathrm{dl}$.

who did not. Neither alcohol consumption nor BMI differed between subjects with or without a low ABI.

\section{Association between CRP and Other Cardiovascular Disease Risk Factors}

The median (interquartile range) of CRP was 0.61 (0.171.37) $\mathrm{mg} / \mathrm{l}$. Table 2 shows the association between CRP value and cardiovascular disease risk factors. The proportion of never smokers was lower in subjects with high CRP, and the proportions of ex-smokers or subjects with hypertension, hypercholesterolemia, diabetes or prior cardiovascular diseases were higher in subjects with the highest CRP level. The proportions of each gender, subjects with low HDL cholesterol or statin users did not differ among the CRP groups. Mean age or alcohol consumption also did not differ among the CRP groups. BMI was lower in the subjects of the lowest CRP group.

\section{OR of Low ABI Was Associated with CRP and Cardio- vascular Disease Risk Factors}

Table 3 shows the results of the multiple logistic regression analysis. Compared with the lowest CRP group, the moder- 
Table 3. Odds Ratio of Low Ankle Brachial Index Associated with Cardiovascular Disease Risk Factors

\begin{tabular}{|c|c|c|c|}
\hline & Odds ratio & $95 \% \mathrm{CI}$ & $p$ \\
\hline Age (5 years) & 1.23 & $0.91-1.67$ & 0.18 \\
\hline Sex $($ male $=1)$ & 1.77 & $0.74-4.23$ & 0.20 \\
\hline Current smoker & 3.10 & $1.16-8.32$ & 0.02 \\
\hline Ex-smoker & 1.51 & $0.62-3.71$ & 0.50 \\
\hline $\begin{array}{l}\text { Alcohol consumption } \\
\text { ( } 23 \text { g/day) }\end{array}$ & 1.01 & $0.79-1.29$ & 0.97 \\
\hline Body mass index $\left(\mathrm{kg} / \mathrm{m}^{2}\right)$ & 0.89 & $0.80-0.99$ & 0.03 \\
\hline Hypertension & 4.29 & $1.60-11.50$ & $<0.01$ \\
\hline Diabetes & 3.73 & $1.82-7.66$ & $<0.01$ \\
\hline Hypercholesterolemia & 1.10 & $0.56-2.14$ & 0.79 \\
\hline Low HDL cholesterol & 3.39 & $1.69-6.81$ & $<0.01$ \\
\hline Use of statin drugs & 3.51 & $1.71-7.19$ & $<0.01$ \\
\hline $\begin{array}{l}\text { History of cardiovascular } \\
\text { diseases }\end{array}$ & 1.74 & $0.89-3.40$ & 0.10 \\
\hline \multicolumn{4}{|l|}{ CRP } \\
\hline$-0.9 \mathrm{mg} / \mathrm{l}$ & 1.00 & & \\
\hline $1-2.9 \mathrm{mg} / \mathrm{l}$ & 2.20 & $1.10-4.41$ & 0.03 \\
\hline $3-\mathrm{mg} / \mathrm{l}$ & 2.06 & $0.90-4.75$ & 0.09 \\
\hline$p$ for trend & & & 0.03 \\
\hline $\begin{array}{l}\text { CRP log-transformed } \\
\text { (continuous) }\end{array}$ & 2.15 & $1.21-3.82$ & $<0.01$ \\
\hline
\end{tabular}

CI, confidence interval. Hypertension: home systolic blood pressure (BP) was at least $135 \mathrm{mmHg}$ and/or home diastolic BP was at least $85 \mathrm{mmHg}$, or they were using antihypertensive agents. Diabetes: non-fasting blood glucose level was at least 200 $\mathrm{mg} / \mathrm{dl}$, or if they currently used antidiabetic medication. Hypercholesterolemia: level of total cholesterol was at least $220 \mathrm{mg} / \mathrm{dl}$, or they currently used non-statin lipid-lowering agents. Low HDL cholesterol: level of high density lipoprotein cholesterol below $40 \mathrm{mg} / \mathrm{dl}$.

ate CRP group and the highest CRP group had a two-fold higher OR. The $p$-value for the trend across CRP groups was statistically significant ( $p=0.03$ ). Furthermore, when we repeated the regression by treating the log-transformed CRP value as a continuous variable, a positive trend between logtransformed CRP and low ABI was also observed $(p<0.01)$.

The following relationships between other cardiovascular disease risk factors and low ABI were found (Table 3). Current smoking, low HDL cholesterol, and history of hypertension, diabetes and statin use were related significantly to low $\mathrm{ABI}$. Lower BMI as a continuous variable was significantly related to low ABI. A history of cardiovascular diseases tended to be related to lower ABI, although the relationship was only marginally significant. Age, sex, alcohol consumption and history of hypercholesterolemia were not significantly related to low ABI.

When we excluded the subjects who were statin users, a significant positive relationship between log-transformed CRP and low ABI remained $(p<0.01)$.

\section{Association of OR of Low ABI with a Combination of Cardiovascular Disease Risk Factors and CRP}

Table 4 shows that the OR of low ABI was associated with the combination of a number of cardiovascular disease risk factors and CRP. In this analysis, according to the results of Table 3, we treated hypertension, diabetes, current smoking and low HDL cholesterol as dichotomous cardiovascular disease risk factors. We also treated the subjects with a CRP level higher than $1.0 \mathrm{mg} / \mathrm{l}$ as high-CRP subjects, because both CRP groups above $1.0 \mathrm{mg} / \mathrm{l}$ showed a similar association with low ABI.

Irrespective of the number of cardiovascular disease risk factors, a higher CRP level was related to a higher risk of low ABI ( $p$ for interaction $=0.70$ ). Even among the subjects without high CRP levels, the clustering of cardiovascular disease risk factors was related to low ABI. In a multiple logistic regression that included as covariates sex, age, BMI, statin use, and history of cardiovascular disease, the OR for low ABI, compared to 0-1 risk factors, was 5.79 (95\% confidence interval $[\mathrm{CI}]: 2.99-11.20)$ for 2 risk factors and 17.45 (95\% CI: 6.78-49.91) for 3 or more risk factors; the OR for CRP $>1.0 \mathrm{mg} / \mathrm{l}$ was independently 2.10 (95\% CI: 1.13-3.88) compared to the lower CRP values.

\section{Discussion}

In this study, we have demonstrated that, in Japan, CRP is related to low ABI independently of other cardiovascular disease atherosclerosis risk factors, and also reconfirmed the impact of the clustering of traditional cardiovascular disease risk factors on low ABI among the Japanese population.

CRP is a circulating acute-phase reactant that is increased many-fold during the inflammatory response to tissue injury or infection. CRP is synthesized primarily in the liver and its release is stimulated by interleukin 6 and other proinflammatory cytokines. This protein has received substantial attention in recent years as a promising biological predictor of atherosclerotic disease (38). In Western countries, some prospective studies have investigated the relationship between CRP and cardiovascular diseases, including PAD $(1-14,21,22)$.

However, no studies have investigated the relationship between CRP and PAD in Japan, and only a few studies have investigated the relationship between PAD and classical factors in a large sample $(23,24)$.

Shinozaki et al. reported the relationship between low $\mathrm{ABI}(\mathrm{ABI}<1.0)$ and cardiovascular disease risk factors among 446 male workers (23). Multiple logistic regression analyses for low ABI showed that low BMI, high SBP, and current smoking were related positively to low $\mathrm{ABI}$ and current drinking was related negatively to low $\mathrm{ABI}$.

Cui et al. reported the relationship between low ABI $(\mathrm{ABI}<0.9)$ and cardiovascular disease risk factors among 1,219 elderly men (24). They found that low BMI, hyperten- 
Table 4. Odds Ratio of Low ABI Associated with a Combination of Number of Cardiovascular Disease Risk Factors and CRP

\begin{tabular}{|c|c|c|c|c|c|c|}
\hline \multirow{2}{*}{ Numbers of risk factors } & \multicolumn{3}{|c|}{ CRP $(-0.9 \mathrm{mg} / \mathrm{l})$} & \multicolumn{3}{|c|}{ CRP $(1.0 \mathrm{mg} / \mathrm{l}-)$} \\
\hline & Odds ratio & $95 \% \mathrm{CI}$ & $p$ & Odds ratio & $95 \% \mathrm{CI}$ & $p$ \\
\hline $0-1$ & 1.00 & & & 1.91 & $0.74-4.92$ & 0.18 \\
\hline 2 & 5.74 & $2.39-13.80$ & $<0.01$ & 11.21 & $4.46-28.20$ & $<0.01$ \\
\hline $3-$ & 12.46 & $2.89-53.69$ & $<0.01$ & 42.40 & $12.72-141.17$ & $<0.01$ \\
\hline
\end{tabular}

ABI, ankle brachial systolic blood pressure (BP) index; CRP, C reactive protein; CI, confidence interval. Risk factors: hypertension: home systolic BP was at least $135 \mathrm{mmHg}$ and/or home diastolic BP was at least $85 \mathrm{mmHg}$, or they were using antihypertensive agents; diabetes: non-fasting blood glucose level was at least $200 \mathrm{mg} / \mathrm{dl}$, or if they currently used antidiabetic medication; current smoking; low high density lipoprotein (HDL) cholesterol: level of HDL cholesterol below $40 \mathrm{mg} / \mathrm{dl}$; adjusted for sex, age, body mass index, statin using and history of cardiovascular diseases.

sion, low HDL cholesterol, history of stroke, major electrocardiogram abnormality, and current smoking were significantly related to low ABI.

Our results were mostly consistent with these reports, but in our study, unlike those of Shinozaki et al. (23) and Cui et al. (24), diabetes was related independently and significantly to low ABI.

Because statins affect the CRP level $(34,35)$, we treated statin use as an independent variable. In this study we also found that statin use was related to low ABI. These relationships might have been observed because the statins were used specifically to treat PAD or because the statin users were those with the highest pre-treatment serum cholesterol.

These risk factors, i.e., low BMI, hypertension, low HDL cholesterol, and current smoking, have also been associated with low ABI among Western subjects (39-41). Therefore, in this study, we confirmed that similar correlations of low $\mathrm{ABI}$ and cardiovascular disease risk factors exist among Japanese subjects and subjects in Western countries.

The CRP level was related to low ABI independently of these cardiovascular disease risk factors, and the relationship also remained when we excluded the statin users.

Since Albert et al. reported that CRP level is related positively to risk clustering (37), we attempted to investigate the relationship between $\mathrm{ABI}$ associated with a combination of number of cardiovascular disease risk factors and CRP. The results also showed that $\mathrm{CRP}$ was related independently to low ABI independent of the number of traditional cardiovascular diseases. Furthermore, the results confirmed the importance of clustering traditional cardiovascular disease risk factors; even those subjects who had multiple risk factors without high CRP levels had a higher OR. Measuring CRP together with traditional cardiovascular disease risk factors may improve our ability to identify individuals with low ABI in the Japanese population.

Our study had some limitations. First, most of the participants were sufficiently active and healthy to participate in the survey; therefore, we have likely underestimated the prevalence of low ABI. Secondly, since this was a cross-sectional study, we cannot conclude that CRP causes PAD or that atherosclerosis leads to higher CRP. Therefore, a prospective study should be undertaken to confirm the relationship between CRP and low ABI in the Japanese population.

In conclusion, we have demonstrated that CRP is related to low ABI. This is the first study to clarify the relationship between CRP and low ABI among Japanese elderly.

\section{Acknowledgements}

The authors are grateful to all the participants of the Tsurugaya Project, and to Yoshiko Nakata, Mika Wagatsuma, and Reiko Taneichi for their secretarial assistance. The authors also thank Professor David Jacobs for his valuable comments on this paper.

\section{References}

1. Kuller LH, Tracy RP, Shaten J, Meilahn EN: Relation of Creactive protein and coronary heart disease in the MRFIT nested case-control study: Multiple Risk Factor Intervention Trial. Am J Epidemiol 1996; 144: 537-547.

2. Tracy RP, Lemaitre RN, Psaty BM, et al: Relationship of C-reactive protein to risk of cardiovascular disease in the elderly: results from the Cardiovascular Health Study and the Rural Health Promotion Project. Arterioscler Thromb Vasc Biol 1997; 17: 1121-1127.

3. Koenig W, Sund M, Frohlich M, et al: C-reactive protein, a sensitive marker of inflammation, predicts future risk of coronary heart disease in initially healthy middle-aged men: results from the MONICA (Monitoring Trends and Determinants in Cardiovascular Disease) Augsburg Cohort Study, 1984 to 1992. Circulation 1999; 99: 237-242.

4. Mendall MA, Strachan DP, Butland BK, et al: C-reactive protein: relation to total mortality, cardiovascular mortality and cardiovascular risk factors in men. Eur Heart $J$ 2000; 21: $1584-1590$.

5. Ridker PM, Hennekens CH, Buring JE, Rifai N: C-reactive protein and other markers of inflammation in the prediction of cardiovascular disease in women. $N$ Engl J Med 2000; 342: 836-843.

6. Danesh J, Whincup P, Walker M, et al: Low grade inflammation and coronary heart disease: prospective study and updated meta-analyses. BMJ 2000; 321: 199-204.

7. Ridker PM, Rifai N, Clearfield M, et al, Air Force/Texas Coronary Atherosclerosis Prevention Study Investigators: 
Measurement of C-reactive protein for the targeting of statin therapy in the primary prevention of acute coronary events. N Engl J Med 2001; 344: 1959-1965.

8. Ridker PM, Stampfer MJ, Rifai N: Novel risk factors for systemic atherosclerosis: a comparison of C-reactive protein, fibrinogen, homocysteine, lipoprotein (a), and standard cholesterol screening as predictors of peripheral arterial disease. JAMA 2001; 285: 2481-2485.

9. Albert CM, Ma J, Rifai N, Stampfer MJ, Ridker PM: Prospective study of C-reactive protein, homocysteine, and plasma lipid levels as predictors of sudden cardiac death. Circulation 2002; 105: 2595-2599.

10. Ridker PM, Rifai N, Rose L, Buring JE, Cook NR: Comparison of C-reactive protein and low-density lipoprotein cholesterol levels in the prediction of first cardiovascular events. N Engl J Med 2002; 47: 1557-1565.

11. Pradhan AD, Manson JE, Rossouw JE, et al: Inflammatory biomarkers, hormone replacement therapy, and incident coronary heart disease: prospective analysis from the Women's Health Initiative observational study. JAMA 2002; 288: 980-987.

12. Ridker PM: High-sensitivity C-reactive protein and cardiovascular risk: rationale for screening and primary prevention. Am J Cardiol 2003; 92: 17K-22K.

13. Ridker PM: Clinical application of C-reactive protein for cardiovascular disease detection and prevention. Circulation 2003; 107: 363-369.

14. Danesh J, Wheeler JG, Hirschfield GM, et al: C-reactive protein and other circulating markers of inflammation in the prediction of coronary heart disease. N Engl J Med 2004; 350: $1387-1397$.

15. Vogt MT, Cauley JA, Newman AB, Kuller LH, Hulley SB: Decreased ankle/arm blood pressure index and mortality in elderly women. JAMA 1993; 270: 465-469.

16. Vogt MT, McKenna M, Anderson SJ, Wolfson SK, Kuller LH: The relationship between ankle-arm index and mortality in older men and women. J Am Geriatr Soc 1993; 41: 523-530.

17. Newman AB, Shemanski L, Manolio TA, et al: Ankle-arm index as a predictor of cardiovascular disease and mortality in the Cardiovascular Health Study: the Cardiovascular Health Study Group. Arterioscler Thromb Vasc Biol 1999; 19: 538-545.

18. Tsai AW, Folsom AR, Rosamond WD, Jones DW: Anklebrachial index and 7-year ischemic stroke incidence: the ARIC study. Stroke 2001; 32: 1721-1724.

19. Abbott RD, Rodriguez BL, Petrovitch H, et al: Anklebrachial blood pressure in elderly men and the risk of stroke: the Honolulu Heart Program. J Clin Epidemiol 2001; 54: 973-978.

20. Schroll M, Munck O: Estimation of peripheral arteriosclerotic disease by ankle blood pressure measurements in a population study of 60-year-old men and women. J Chron Dis 1981; 34: 261-269.

21. Ridker PM, Cushman M, Stampfer MJ, Tracy RP, Hennekens $\mathrm{CH}$ : Plasma concentration of C-reactive protein and risk of developing peripheral vascular disease. Circulation 1998; 97: 425-428.

22. Van Der Meer IM, De Maat MP, Hak AE, et al: C-reactive protein predicts progression of atherosclerosis measured at various sites in the arterial tree: the Rotterdam Study. Stroke 2002; 33: 2750-2755.

23. Shinozaki T, Hasegawa T, Yano E: Ankle-arm index as an indicator of atherosclerosis: its application as a screening method. J Clin Epidemiol 1998; 51: 1263-1269.

24. Cui R, Iso H, Yamagishi K, et al: Ankle-arm blood pressure index and cardiovascular risk factors in elderly Japanese men. Hypertens Res 2003; 26: 377-382.

25. Ohmori K, Ebihara S, Kuriyama S, et al: The relationship between body mass index and a plasma lipid peroxidation biomarker in an older, healthy Asian community. Ann Epidemiol 2005; 15: 80-84.

26. Hozawa A, Ebihara S, Ohmori K, et al: Increased plasma 8-isoprostane levels in hypertensive subjects: the Tsurugaya Project. Hypertens Res 2004; 27: 557-561

27. Imai Y, Satoh H, Nagai K, et al: Characteristics of a community based distribution of home blood pressure in Ohasama, a northern part of Japan. J Hypertens 1993; 11: 1441-1449.

28. Ledue TB, Weiner DL, Sipe JD, Poulin SE, Collins MF, Rifai N: Analytical evaluation of particle-enhanced immunonephelometric assays for C-reactive protein, serum amyloid A and mannose-binding protein in human serum. Ann Clin Biochem 1998; 35: 745-753.

29. Yamashina A, Tomiyama H, Takeda K, et al: Validity, reproducibility, and clinical significance of noninvasive brachial-ankle pulse wave velocity measurement. Hypertens Res 2002; 25: 359-364.

30. Ohnishi H, Saitoh S, Takagi S, et al: Pulse wave velocity as an indicator of atherosclerosis in impaired fasting glucose: the Tanno and Sobetsu study. Diabetes Care 2003; 26: 437-440.

31. Okamura T, Moriyama Y, Kadowaki T, Kanda H, Ueshima $\mathrm{H}$ : Non-invasive measurement of brachial-ankle pulse wave velocity is associated with serum C-reactive protein but not with $\alpha$-tocopherol in Japanese middle-aged male workers. Hypertens Res 2004; 27: 173-180.

32. Chobanian AV, Bakris GL, Black HR, et al: The seventh report of the Joint National Committee on Prevention, Detection, Evaluation, and Treatment of High Blood Pressure: the JNC 7 report. JAMA 2003; 289: 2560-2571.

33. European Society of Hypertension-European Society of Cardiology Guidelines Committee: 2003 European Society of Hypertension-European Society of Cardiology guidelines for the management of arterial hypertension. J Hypertens 2003; 21: 1011-1053.

34. Albert MA, Danielson E, Rifai N, Ridker PM, PRINCE Investigators: Effect of statin therapy on C-reactive protein levels: the pravastatin inflammation/CRP evaluation (PRINCE): a randomized trial and cohort study. JAMA 2001; 286: 64-70.

35. Ridker PM, Rifai N, Lowenthal SP: Rapid reduction in Creactive protein with cerivastatin among 785 patients with primary hypercholesterolemia. Circulation 2001; 103: 1191-1193.

36. Yamada S, Gotoh T, Nakashima Y, et al: Distribution of serum C-reactive protein and its association with atherosclerotic risk factors in a Japanese population: Jichi Medical School Cohort Study. Am J Epidemiol 2001; 153: 1183-1190. 
37. Albert MA, Glynn RJ, Ridker PM: Plasma concentration of C-reactive protein and the calculated Framingham Coronary Heart Disease Risk Score. Circulation 2003; 108: 161-165.

38. Hackam DG, Anand SS: Emerging risk factors for atherosclerotic vascular disease: a critical review of the evidence. JAMA 2003; 290: 932-940.

39. Meijer WT, Grobbee DE, Hunink MG, Hofman A, Hoes AW: Determinants of peripheral arterial disease in the el- derly: the Rotterdam study. Arch Intern Med 2000; 160: 2934-2938.

40. Murabito JM, Evans JC, Nieto K, Larson MG, Levy D, Wilson PW: Prevalence and clinical correlates of peripheral arterial disease in the Framingham Offspring Study. Am Heart J 2002; 143: 961-965.

41. McDermott MM, Green D, Greenland P, et al: Relation of levels of hemostatic factors and inflammatory markers to the ankle brachial index. Am J Cardiol 2003; 92: 194-199. 